Individual questions were further analysed to ascertain if there were particular drugs causing difficulty. Analysis showed that a question on potassium chloride yielded low pre-assessment scores of $21 \%$ and $39 \%$ respectively for MMUH Doctors and Nurses and $45 \%$ and $20 \%$ for Peamount. Although both disciplines improved, this demonstrated a need for further training with this drug.

Conclusions The e-learning programme showed a significant increase in user knowledge, in both hospitals, for all disciplines. These results are very encouraging given the differences between the institutions, grades of staff and experience. The results do not stem from a 'specific teacher effect' and therefore are reproducible in multiple sites.

No conflict of interest.

\section{GRP-015 AN OVERVIEW OF HOSPITAL PHARMACEUTICAL EXPENDITURE IN GREECE OVER THE LAST TWO YEARS}

doi:10.1136/ejhpharm-2013-000276.015

'K Nikou, ${ }^{2} \mathrm{~K}$ Perdikouri, ${ }^{3} \mathrm{M}$ Katharaki. 'General Hospital of Chest Diseases "SOTIRIA", Pharmacy, Athens, Greece; 'Spiliopoulio Hospital, Pharmacy, Athens, Greece; ${ }^{3} G e n e r a l$ Hospital "Alexandra", Nursing Services Division, Athens, Greece

Background Under the burden of the economic crisis in Greece, a series of cost containment measures for hospitals' operating costs have been implemented, with the emphasis put on limiting the money spent on medicines purchasing.

Purpose To review the extent to which the target of reducing hospital pharmaceutical expenses has been achieved along with reporting the changes, as far as the 'in hospital' use of generic medicine is concerned.

Materials and Methods Financial data from 136 Greek public hospitals, as officially reported in ESY.net database, were collected and compared for the years of interest. The financial data were selected with respect to the cost of purchasing medicines and nonpharmaceutical material, while other operational costs were omitted. Results The cost of purchasing medicines constantly accounts for a high percentage of a hospital's budget for supplies $(51 \%, 53 \%$ and $56 \%$ for 2010, 2011 and 2012 respectively). An overall decrease in pharmaceutical expenditures was achieved (23\% reduction in 2011, along with a further reduction of $17 \%$ in 2012). Although rates of introducing generic drugs differ among different hospitals, an increase in use of generics was observed (26\% in 2011 and 30\% in 2012). Psychiatric hospitals seem to have better scores compared to paediatric and oncology departments.

Conclusions The 'in hospital' use of generic drugs score is significantly higher compared to that of the Greek market in general $(18 \%)$ and has therefore contributed to the hospitals' attempt to reduce the amount of money spent on medicine supplies. The lower rates of generics' use observed in paediatric hospitals are consistent with the lower possibility for substitution in these cases. Last but not least, when selecting and implementing drug cost management strategies, it is essential that pharmacists remain mindful of patient safety and quality of patient care.

No conflict of interest.

\section{GRP-016 ANALYSIS AND CONSUMPTION OF INNOVATIVE ANTIDIABETIC DRUGS IN PIEDMONT PATIENTS}

\section{doi:10.1136/ejhpharm-2013-000276.016}

V Colombardo, V Besso, R Bona, M Conson, M Nocera, M Sampietro, I Barbato, P Mero, D Piccioni, S Martinetti. Hospital Pharmacy, ASL AT, Asti, Italy

Background The increase in deaths due to diabetes records a trend in growth and the OSMED National Report of 2011 highlights a prescription shift towards the high-cost innovative drugs for the treatment of type II diabetes mellitus (DMII). This is subject to intensive monitoring by the health ministry.

In the management of diabetic patients, the guidelines suggest an early intensive therapeutic intervention and the pursuit of a personal glycaemic target for avoiding hypoglycaemic episodes, which are possibly responsible for the increased risk of developing cardiovascular episodes.

Purpose To analyse the population, consumption and type of innovative diabetic drugs used in the Piedmont region; this is to put a value on the type of treatment used for DMII, because the advantages of innovative therapy must be valued too.

Materials and Methods In the first step the incidence of DMII in Piedmont was valued by analysing data from the regional diabetic database during the period 2007-2012. Dipeptidyl peptidase 4 inhibitors (sitagliptin and vildagliptin alone or in association with metformin and saxagliptin), thiazolidinediones (pioglitazone alone or in association with metformin), glucagon-like peptide 1 (exenatide, liraglutide), insulin glargine and detemir were considered innovative drugs. Consumption and type of drugs were analysed in terms of the defined daily dose/1000 inhabitants/day (DDD) over a six-month period in 2012 using regional databases of prescriptions which enabled us to access population data. 2012 data were compared with 2011.

Results The first striking finding is the increase in the incidence of DMII, $1.70 \%$ in five years, which corresponds to 80,327 patients. Focusing on the population treated with innovative drugs revealed that $21 \%(61,679 / 294,590)$ of diabetic patients are 65 years old and far more males than females are affected (respectively $53.87 \%$ vs. $46.13 \%$ ). The drug most used is insulin glargine with $43.84 \%$ of total consumption, another $25.08 \%$ use DPP4 inhibitors alone or in association, $20.05 \%$ use pioglitazone alone or in association, $9.02 \%$ use glucagon-like peptide 1 and $2.01 \%$ use insulin detemir. The comparison with the same period of 2011 highlights the increased consumption of innovative drugs in Piedmont, 23\% (8.97 DDD in 2012 vs. 6.91 DDD in 2011) while Italian data record an increase of $5 \%$ (15.69 DDD vs. 14.87 in 2011).

Conclusions Increased consumption of these drugs suggests that medical prescriptions could maybe move on innovative therapeutic molecules. It is important that clinicians discuss and compare the data analysis shown above with medical management guidelines, with the aim of estimating the genuine advantages of innovative drugs in terms of compliance, reduction in adverse reactions and increased quality of life.

No conflict of interest.

\section{GRP-017 ANALYSIS AND PREVENTION OF MUSCULOSKELETAL DISORDERS IN A HOSPITAL STERILISATION UNIT}

doi:10.1136/ejhpharm-2013-000276.017

L Tortolano, I Becamel, D Briand, F Vincent. Hopital Européen Georges Pompidou, Paris, France

Background Musculoskeletal disorders (MSDs) are problems caused by the poor ergonomic design of work stations. The daily work of sterilisation, especially carrying heavy loads, carries a risk of developing MSDs.

Our sterilisation unit, certified ISO 9001, tries to improve the working environment including the ergonomics of work stations.

Purpose To establish an inventory of fixtures and to suggest preventive measures in order to limit the appearance of those disorders.

Materials and Methods The whole of the sterilisation unit workforce was interviewed about any pain, physical effort and nonergonomic situations that they routinely face during their daily work. 